\title{
Highly-Sensitive Magnetic Tunnel Junction Based Flow Cytometer
}

\author{
Selma Amara ${ }^{1}$, Ride Bu ${ }^{1,2}$, Meshal Alawein ${ }^{1}$, Nouf Alsharif ${ }^{1}$, Mohammed Asadullah Khan ${ }^{1}$, Yan Wen ${ }^{1}$, Xixiang Zhang ${ }^{1}$, Jurgen Kosel ${ }^{1}$, Hossein Fariborzi
}

${ }^{1}$ King Abdullah University of Science and Technology, 4700 Thuwal, Saudi Arabia

${ }^{2}$ School of Physics. Shandong University, Jinan 250000, China

\begin{abstract}
Flow cytometers are important instruments for biological and biomedical analyses. These instruments are large and expensive, and researchers are continuously striving to come up with smaller, cheaper, and more energy-efficient flow cytometers. In this work, we present a highly-sensitive magnetic tunnel junction (MTJ) based flow cytometer. An externally magnetized magnetic beads labeling cells were placed above an MTJ sensor that can measure the stray field surrounding the beads. It was found that each time labeled cells pass through the sensitive area of the sensor, a peak of signal was observed. The results demonstrate a novel MTJ based flow cytometer design approach for accurate detection of magnetically labeled cells.
\end{abstract}

Keywords-Biosensor, magnetic tunnel junction, PDMS microchannel, magnetic bead, flow cytometer.

\section{INTRODUCTION}

The advancements in spintronic sensors and their applications to biomedical devices has given rise to a new family of highly-sensitive and small biomedical sensors [1-7]. A typical spintronic sensor nowadays is based on a magnetic tunnel junction (MTJ) comprised of a thin insulating layer separated by two ferromagnetic layers. Such tunneling structures exhibit the tunnel magnetoresistance (TMR) effect, which is a result of the change in electrical resistance between parallel and antiparallel states. Compared with giant magnetoresistive sensors, TMR sensors show higher sensitivity, a key requirement for the efficient detection of small magnetic fields typically found in biomedical applications.

Flow cytometers are essential instruments for cell-analysis and diagnostic applications. A cytometer instrument is large, complex, and expensive. These limitations have encouraged researchers to employ new micro-fabrication and microfluidics control technologies to achieve cleaner, smaller, more efficient and reliable microfluidic cytometers. One approach to tackle the above problem could be through the use of spintronic sensors [8].

In this paper, we present simulation, fabrication, and characterization of a highly-sensitive MTJ-based flow cytometer for the detection of magnetically labeled cells.

\section{MTJ BASED Flow Cytometer}

\section{A. Device concept}

The proposed sensor is shown in Fig. 1 (a) and consists of one MTJ pillar and a polydimethylsiloxane (PDMS) microchannel. Fig. 1 (b) illustrates the sensing concept with superparamagnetic beads, which are magnetized by an external field, that are labeling the cells. The target cells are human embryonic kidney cells 293 (HEK 293), which are widely used in cell biology and biotechnology. The stray fields of the bead can be sensed by the MTJ, if the magnetic bead is within the sensing region. The MTJ sensors used are magnetized in-plane, and sensitive in the $y$-direction corresponding to the sensor easy axis. When a cell labeled by magnetic beads passes over the MTJ, the resistance of the MTJ changes, and this variation is inferred by measuring the variation of the MTJ output voltage, $\Delta V$.

(a)

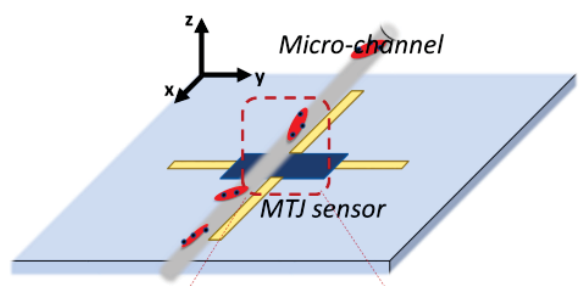

(b)

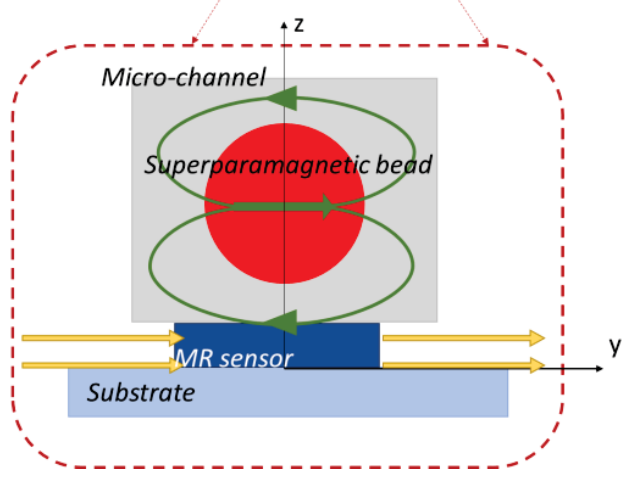

Fig. 1. (a) Magnetic biosensor system consisting of one pillar of MTJ sensor, and a microchannel for labeled cells. The external magnetic field is applied in the $y$-direction. (b) Cross-section view of MTJ sensor. An external magnetic field magnetizes the superparamagnetic bead labeling the cell. The stray field of the magnetic bead is detected by the MTJ sensor. The yellow lines represent the external applied magnetic field.The green lines represent the stray field of the magnetic bead.

\section{B. Simulation}

A magnetic bead has an equal probability to attach to any location on the target cell. To simplify calculations, the beadcell compound is assumed to be spherical, as shown in Fig 2. The diameter of the HEK 293 and the magnetic bead are $8 \mu \mathrm{m}$ and $2.8 \mu \mathrm{m}$, respectively. The stray magnetic field of the bead is given by 


$$
\mathbf{H}_{\text {bead }}=\frac{1}{4 \pi}\left[\frac{3(\mathbf{m} \cdot \mathbf{r}) \mathbf{r}}{|\mathbf{r}|^{5}}-\frac{\mathbf{m}}{|\mathbf{r}|^{3}}\right]
$$

where $\mathbf{m}$ is the magnetic moment of the bead, calculated using $\mathbf{m}=(4 \pi / 3) R^{3} \mathbf{M}$, where $\mathbf{M}=\left(1 / 3+1 / \chi_{m}\right)^{-\mathbf{1}} \mathbf{H}_{\text {ext }}$ and $\mathbf{H}_{\text {ext }}$ is the external magnetic field. Here $R$ and $\chi_{m}$ are the radius and the magnetic susceptibility of the magnetic bead. Since the MTJ is more sensitive to magnetic fields in the $y$ direction, we considered only $H_{b e a d, y}$. The average value of $H_{\text {bead }, y}$ is given by [8]

$$
\left(H_{\text {bead }, y}\right)_{\text {avg }}=\int_{-l / 2}^{l / 2} \int_{-h / 2}^{h / 2} H_{\text {bead }, y} d y d x,
$$

where $h$ and $l$ are the width and length of the MTJ, respectively. The output voltage of the MTJ is calculated considering a linear response of the sensor to an external magnetic field [9]

$$
\Delta V=-S I_{M T J}\left(H_{\text {bead }, y}\right)_{a v g}
$$

where $S$ is the sensor sensitivity and $I_{M T J}$ is the sensing current. The sensor sensitivity is obtained from the MTJ transfer characteristics.

Here, we used Eqs. (2) and (3) to obtain the sensor output voltage shown in Fig. 3. The simulations are done for a $8 \mu \mathrm{m}$ cell-bead compound magnetized by a magnetic field in the $y$ direction. The cell-bead compound flows over the MTJ sensor in three different directions: $(0, y, 5),(20, y, 10)$ and $(50, y, 20)$.

In order to conform to experiment result, the following parameters were used: $h=8 \mu \mathrm{m}, l=16 \mu \mathrm{m}, I_{M T J}=\frac{V}{R_{R e f}+R_{M T J}}$, $\mathrm{V}=1 \mathrm{mV}$ is the applied voltage, $R_{M T J}=10 \Omega, R_{R e f}=50 \Omega$ (the reference resistance of the lock-in amplifier used), $\chi_{m}=0.79$, $R=1.4 \mu \mathrm{m}, S=1.422 \Omega \cdot \mathrm{Oe}^{-1}$, and $H_{\text {ext }}=30 \mathrm{Oe}$.

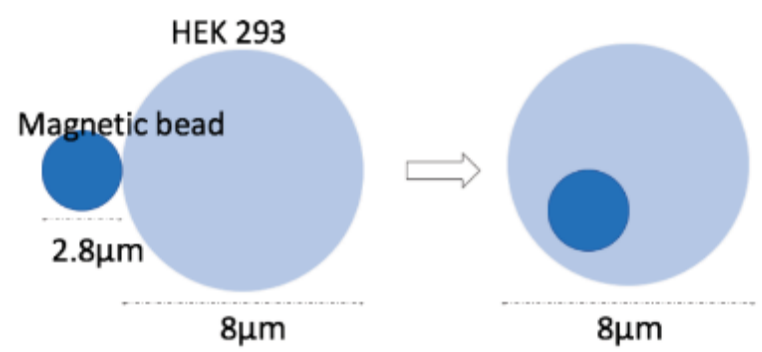

Fig. 2. The HEK 293 is labeled by a magnetic bead. The magnetic bead goes into the HEK 293 cell.

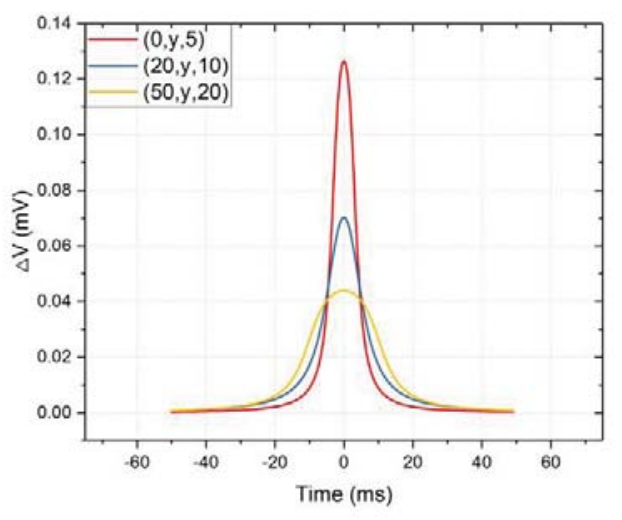

Fig. 3. Simulated signals for cell-bead compound magnetized in the $y$ direction. The cell-bead flows at three different directions: $(0, y, 5),(20, y, 10)$, and $(50, y, 20)$.

\section{MATERIAL AND METHODS}

\section{A. Fabrication and characterization}

We designed and fabricated the MTJ sensors presented in Fig. 4. Each sensor consists of one elliptic pillar $\left(16 \times 8 \mu \mathrm{m}^{2}\right)$. MTJ multilayer films were deposited using a magnetron sputtering system (Singulus Rotaris) on thermally oxidized $\mathrm{Si}$ wafers. The MTJ stack used in this study (see Fig4. (a) ) had the following layer structure, where a value in parenthesis represents the thickness in $\mathrm{nm}$ : $\mathrm{Si} / \mathrm{SiO} / \mathrm{Ru}(3) / \mathrm{Ta}(8) / \mathrm{Ru}(3) / \mathrm{Ta}(8) / \mathrm{Ru}(3) / \mathrm{MnIr}(8) / \mathrm{Co}_{70} \mathrm{Fe}_{30}(2.3) /$ $\mathrm{Ru}(0.85) / \mathrm{Co}_{60} \mathrm{Fe}_{20} \mathrm{~B}_{20}(2.4)$ “pinned layer"/ $\mathrm{MgO}(1.53)$ "oxide barrier" / $\mathrm{Co}_{60} \mathrm{Fe}_{20} \mathrm{~B}_{20}(1.45)$ "free layer"/ $\mathrm{Ru}(3) / \mathrm{Ta}(8)$.

The MTJ stack was patterned into micron-sized elliptical devices using standard optical lithography and ion milling. A 150-nm-thick gold layer was deposited over the junction area of each MTJ. After patterning, the samples were annealed at 360 ${ }^{\circ} \mathrm{C}$ for 2 hours at $1.10^{-6}$ Torr in an applied field of $8 \mathrm{kOe}$. An insulator of $50 \mathrm{~nm}$ of $\mathrm{SiO}_{2}$ was sputtered on the MTJ sensor surface to protect it against corrosion.

The magnetoresistance properties of the MTJ sensors were measured at room temperature by a conventional DC four-probe method and current driven Helmholtz coils controlled with LabView. Fig. 4 (c) shows the transfer curve of the MTJ sensor. The resistance of the MTJ sensor changes as the relative orientation of the magnetizations of the two ferromagnetic layers changes. When the magnetizations of the two ferromagnetic layers are parallel, the MTJ sensor shows minimal resistance; when they are antiparallel, the MTJ sensor shows maximal resistance. The magnetization of the bottom layer is pinned by using interface exchange coupling with an antiferromagnetic layer, and the top layer is free to rotate. This structure generates a linear sensor response to external magnetic fields along the sensor's transverse direction. For the MTJ used in our experiment, the TMR ratio is $43.158 \%$ with a sensitivity of $1.422 \Omega \cdot \mathrm{Oe}^{-1}$. The sensor has a linear response in the range [25Oe, 35Oe].

\section{B. Microchannel fabrication}

The microchannel was made of PDMS. The PDMS prepolymer was prepared by mixing the base and curing agent (SYLGARD ® 184, Silicon Elastomer KIT, Dow Corning) in a 
10:1 weight ratio and heating for 1 hour in a $70^{\circ} \mathrm{C}$ thermostat box. The PDMS was $3 \mathrm{~mm}$ thick, and the microchannel was fabricated on the surface. The microchannel was designed using the Corel Draw X5 software. The channel layout was printed on the PDMS surface using a laser (Universal laser system PLS6.150D). The inlets and outlets of the channel were punched through PDMS using a biopsy punch with $1 \mathrm{~mm}$ in diameter.

\section{Assembly of the sensor}

The assembly of the sensor was finalized by bonding the PDMS microchannel to the sputtered wafer surface. All dust and stain were removed to assure a stable bonding between the two surfaces. The wafer and PDMS surfaces were both washed with acetone for two minutes. An $\mathrm{O}_{2}$ plasma treatment was carried out for $40 \mathrm{~s}$ at $60 \mathrm{~mW}$ to produce chemical (siloxane) bonds between the two participating surfaces. The bonding was observed under the microscope to precisely align the channel with the MTJ sensor.

\section{Experiment setup}

For real-time detection of the labeled cells, a measurement setup was established as shown in Fig. 4. The MTJ sensor used in the experiment has a resistance comparable to the contacts resistance. In order to eliminate the influence of lead resistance, four-probe measurement is employed. The sensor has four contact pads; two pads connect the top electrode of the sensor, and the other two pads connect the bottom electrode.

The MTJ sensor was powered by a lock-in amplifier (SR850), which was connected to the top electrode. The lock-in amplifier was also connected to the bottom electrode of the sensor. The analog output from the lock-in amplifier was picked up by a GPIB to USB 2.0 converter (Keysight Agilent 82357B) and tranfered to a computer which was controlled with LabView. A syringe pump (CorSolutions, PeriWave Pump Micro) was used to control the rate of fluid flow through the microchannel (flow rate ranges as $0.1-50 \mathrm{microL} / \mathrm{min}$ ). The cellbead compounds were pumped into the microchannel through microtubes (CorSolutions, 1/32-inch Peek tubing). (a)

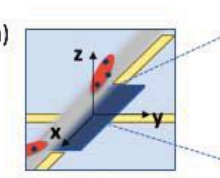
One MTJ sensor

(b)

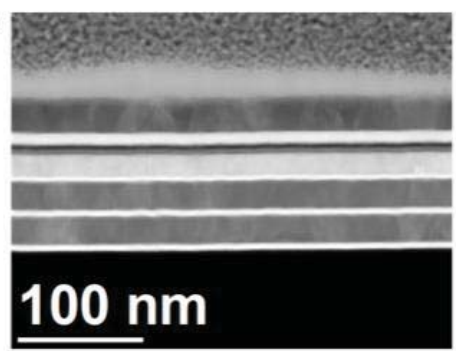

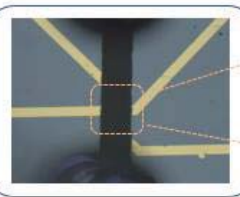
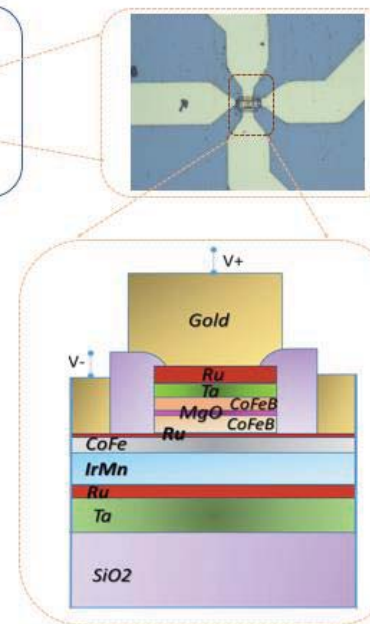

(c)

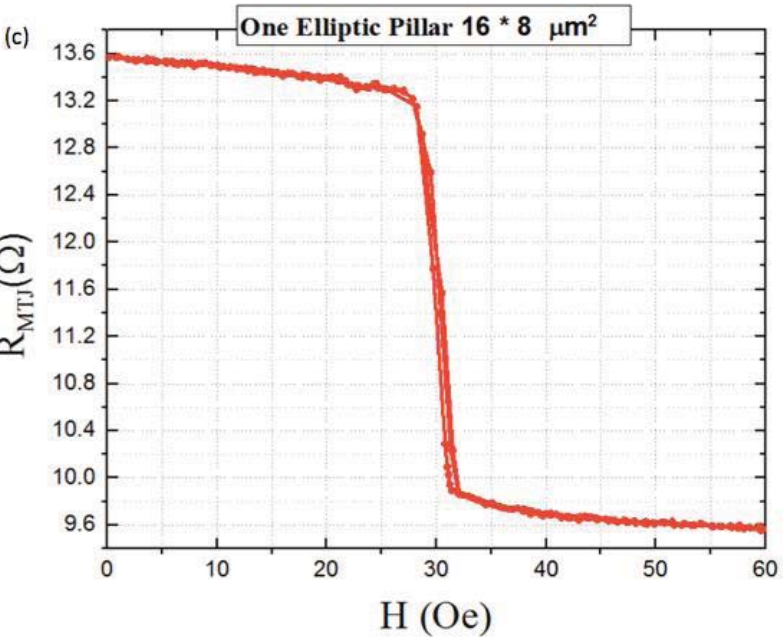

Fig. 4. (a) Zoomed-in view of one biosensor (one MTJ pillar). (b) Transmission Electron Microscopy image of the MTJ multilayer. (c) Device transfer curve. 


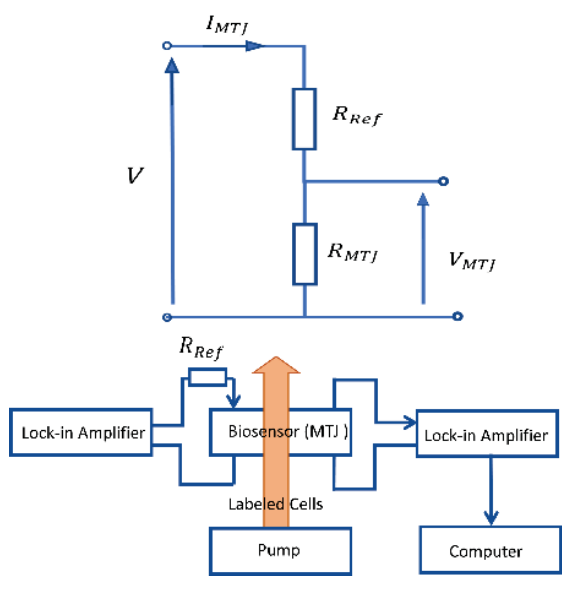

Fig. 5. The biosensor was powered by a lock-in amplifier. Labeled cells were pumped into the microchannel and passed over the MTJ sensor surface. The real-time voltage change was detected by a lock-in amplifier conneted to a computer.

\section{RESUlTS AND DisCUSSION}

All experiments were conducted by applying an AC voltage $\mathrm{V}=1 \mathrm{mV}$ with a frequency of $58 \mathrm{kHz}$. Since the beads are superparamagnetic, an 30 Oe external magnetic field was applied by an electromagnet. $1 \mu \mathrm{L}$ droplet of the cell-bead compounds was pumped across the surface of MTJ sensors for the detection of signals of beads labeled cells.
It is worth noting that the measured flow includes both labelled cells and free beads, and some cells might not be labelled. We leave the beads with cells in a container for one day to make sure that most of the cells are labelled by beads. To improve the system, in the next step of this study, we will add a trapping magnet that will filter free beads. As Fig. 6 (a) shows, when a labeled cell passes over the MTJ surface, an output voltage change is detected. Sharp signals are indicative of labeled cells passing by the sensor. Different signal intensities correspond to the coordinate of cells in the microchannel. The zoomed-in view of the largest signal (between $450 \mathrm{~ms}$ and $500 \mathrm{~ms}$ ) is similar to the simulation result shown in Fig. 3. To confirm that the signal acquired by the SR850 was due to magnetic-labeled cells and measure the background noise, the same experiment was performed with unlabeled cells. The result in Fig. 6 (b) shows that no signal is detected with unlabeled cells. Obviously, all detections in Fig. 6 (a) comes from labeled cells. The data shown in Fig. 6 (b) is used to determine the background noise. The positive and negative threshold of background noise is $+0.02 \mathrm{mV}$ and $-0.02 \mathrm{mV}$. The noise interval $[-0.02,+0.02 \mathrm{mV}]$ is important in the software cell counting process. All values larger than this interval are considered as labeled cells signal.

The largest and smallest signals in the experiment are 0.125 $\mathrm{mV}$ and $0.045 \mathrm{mV}$, respectively. Such values demonstrate the performance of MTJ sensors to detect the small magnetic fields of the beads that label cells. We repeated this measurement 10 times with different sensors design (1 pillar and 10 pillars) and it shows a good repeatability (a)

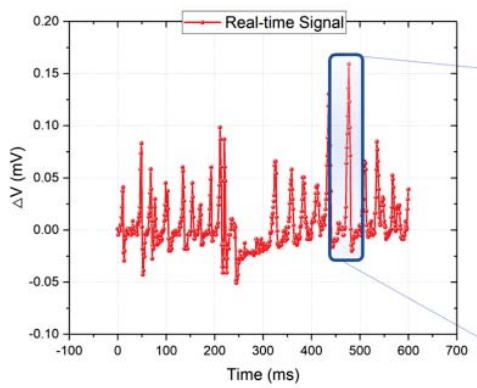

(b)

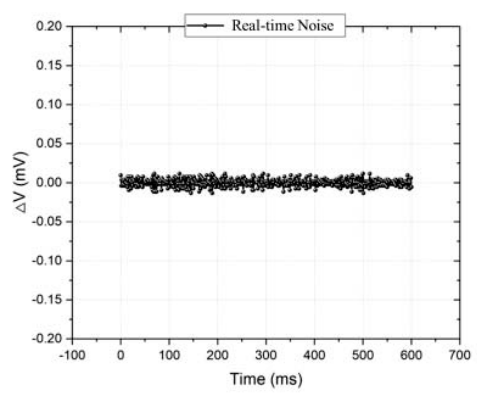

(c)

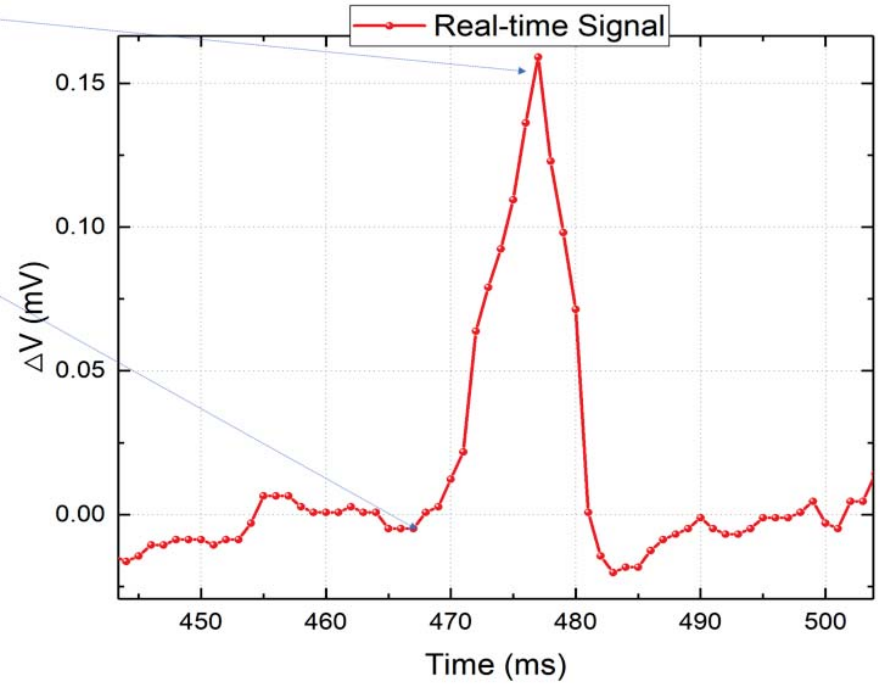

Fig. 6. (a) Real-time voltage change $\Delta V$ indiactes that labeled cells passe over the MTJ sensor surface. (b) Zoomed-in view of the largest signal from 450 to $500 \mathrm{ms,}$ with a voltage output similar to the simulation result shown in Fig. 3. (c) Background noise, when unlabeled cells go through the microchannel. 


\section{CONCLUSION}

In this work we proposed a highly-sensitive MTJ based flow cytometer and demonstrated its functionality for detection of magnetically labeled cells. We fabricated and characterized the proposed device and showed that our biochip cytometer, with dimensions much smaller than ordinary detection instruments, performs well the real-time survey of cells injection for medical treatments or analyses in biomedical studies. The cytometer would be improved by a trapping magnet that would filter free beads.

\section{ACKNOWLEDGMENT}

Research reported in this publication was supported by the King Abdullah University of Science and Technology (KAUST).

\section{REFERENCES}

[1] F. Li and J. Kosel: “An efficient biosensor made of an electromagnetic trap and a magneto-resistive sensor". Biosensors and Bioelectronics, 59, pp. 145-150 (2014).

[2] Wolf, S. A., et al. "Spintronics: a spin-based electronics vision for the future." Science 294.5546 1488-1495, 2001.

[3] B. M. de Boer, J. A. H. M. Kahlman, T. P. G. H. Jansen, H. Duric, and J. Veen, "An integrated and sensitive detection platform for magneto-resistive biosensors," Biosens. Bioelectron., vol. 22, 2366-2370, April 2007.

[4] L. A. Herzenberg, D. Parks, B. Sahaf, O. Perez, M. Roederer, and L. A. Herzenberg, "The History and Future of the Fluorescence Activated Cell Sorter and Flow Cytometry: A View from Stanford," Clin. Chem, vol. 48, pp. 1819-1827, October 2002.

[5] J. Germano, V. Martins, F. Cardoso, T. Almeida, L. Sousa, P. Freitas, and M. Piedade, "A Portable and Autonomous Magnetic Detection Platform for Biosensing," Sensors, 2009, vol. 9, pp. 4119-4137, May 2009.

[6] Andrei Jitariu, Carla Duarte, Susana Cardoso, Paulo P. Freitas, Horia Chiriac. "Numerical Evaluation of Bacterial Cell Concentration by Magnetoresistive Cytometry", IEEE Transactions on Magnetics, vol 53, April 2017.

[7] B. M. de Boer, J. A. H. M. Kahlman, T. P. G. H. Jansen, H. Duric, and J. Veen, “An integrated and sensitive detection platform for magneto-resistive biosensors," Biosens. Bioelectron., vol. 22, 2366-2370, April 2007.

[8] J. Loureiro, P. Z. Andrade, S. Cardoso, C. L. da Silva, J. M. Cabral, and P. P. Freitas, "Magnetoresistive chip cytometer,” Lab Chip, vol. 11, pp. 2255-2261, 2011 .

[9] Jitariu, Andrei, et al. "Numerical Evaluation of Bacterial Cell Concentration by Magnetoresistive Cytometry." IEEE Transactions on Magnetics 53.4 (2017): 1-4. J. Germano, V. Martins, F. Cardoso, T. Almeida, L. Sousa, P. Freitas, and M. Piedade, "A Portable and Autonomous Magnetic Detection Platform for Biosensing," Sensors, 2009, vol. 9, pp. 4119-4137, May 2009. 